\title{
Desafíos del intraoperatorio
}

\author{
MARCELA ÁVILA Á. ${ }^{1}$, CARLOS MARGARIT B. ${ }^{2}$
}

Hospital de Urgencia Asistencia Pública, Instituto de Salud del Trabajador, Universidad de Santiago. Hospital San Juan de Dios, La Serena, Universidad Católica del Norte.

Ya se ha mencionado el gran desafío en el que se puede tornar el manejo anestésico de los grandes quemados. En el artículo Consideraciones Farmacológicas en el Manejo Anestésico del Paciente quemado, de este mismo número, se abordó la farmacología del gran quemado; en la presente revisión, se tratará algunos tópicos del intraoperatorio tales como el manejo de la vía aérea, el control de la temperatura, los accesos vasculares, etc.

\section{Manejo de la vía aérea}

Es frecuente que los pacientes lleguen intubados al pabellón, principalmente durante la fase aguda. Si este no es el caso, se debe considerar que el paciente quemado tiene factores anatómicos, ventilatorios y metabólicos que lo ponen en riesgo de hipoxemia durante la inducción, los que van cambiando dependiendo de la etapa de su evolución clínica ${ }^{1,2,12}$.

En la primera fase, apenas ocurrida la injuria, los pacientes tienen alto consumo de oxígeno y además, durante la laringoscopia, será frecuente encontrar edema de la vía aérea (tanto por las quemaduras como por la reanimación, siendo el peak entre las 8-36 h después de la injuria y dura hasta 4 días), abundantes secreciones y hollín; por otro lado, la desaturación ocurre más fácil en la presencia de daño pulmonar y aumento de la tasa metabólica ${ }^{2,6,13}$.

Posteriormente en su evolución, el mayor desafío son las rigideces producidas por las cicatrices de las quemaduras de cara, cuello y tórax ${ }^{2,9,14}$, a lo que se suma microstomía, fosas nasales anormales y/o pequeñas, estenosis subglótica, traqueomalacia, etc ${ }^{15}$.

En la población pediátrica debemos agregar los desafíos propios de este grupo etario: cabeza y lengua proporcionalmente más grandes, mandíbula más corta y paladar y epiglotis más largos, fosas nasales más pequeñas, glotis más anterior y cefálica, apertura glótica angulada, junto con un mayor riesgo de laringo y broncoespasmo ${ }^{3,15}$.

Kaiser y cols., proponen un algoritmo de manejo de la vía aérea en el paciente gran quemado ${ }^{1}$ que hemos traducido y replicado con autorización de sus autores en la Figura 1.

Antes de decidir el plan de manejo es preciso evaluar la permeabilidad y la compliance de los tejidos involucrados. Si en la palpación de los tejidos del cuello y del espacio submandibular se observa "alta tensión" debido a edema o rigidez, es muy probable que la laringoscopia sea laboriosa dada la dificultad de desplazar la lengua y los tejidos blandos del área submandibular.

Si se sospecha una vía aérea difícil y el paciente es colaborador, se puede planear una fibrobroncoscopia (FBC) con paciente vigil, de lo contrario, se puede intentar igual técnica pero tras una inducción que permita preservar la ventilación espontánea (lo más usado es la ketamina con algún coadyuvante). Si la evaluación con FBC revela que no hay mayor distorsión anatómica que dificulte la intubación se puede proceder al uso de bloqueadores neuromusculares previo test de ventilación con mascarilla.

La ventilación con mascarilla suele verse dificultada, particularmente cuando hay vendajes en la zona cervico-facial. Además, una cánula

Correspondencia a:

Dra. Marcela Ávila Á.

maravila7@gmail.com 


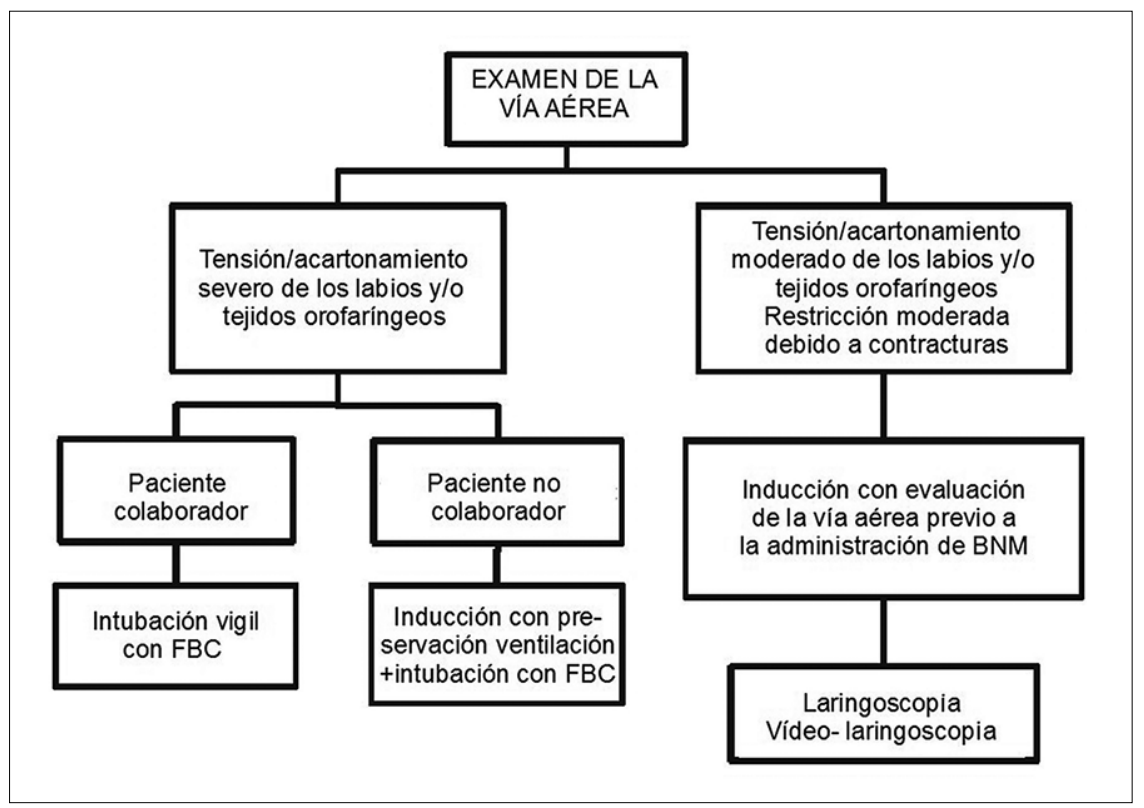

Figura 1. Algoritmo de manejo de la vía aérea en el gran quemado. Adaptado de Kaiser HE. Advances in Perioperative and Critical Care of the Burn Patient. Anesthesia Management of Major Thermal Burn Injuries in Adults. Advances in Anesthesia 2013; 31: 137-161.

orofaríngea puede ser difícil de insertar en un paciente con microstomía. Por otro lado, la posición de olfateo también puede ser difícil de lograr debido a la presencia de vendajes, cicatrices $\mathrm{y}$ rigideces ${ }^{1-3,6,14}$.

La intubación de la tráquea puede ser oral, nasal o transtraqueal, dependiendo del sitio quirúrgico, de la disponibilidad de cada ruta y de la urgencia del procedimiento. La intubación orotraqueal (IOT) se puede realizar con laringoscopia directa, videolaringoscopia, estilete luminoso y FBC, entre otras técnicas ${ }^{1,2}$.

La laringoscopia suele dificultarse o incluso tornarse imposible en la presencia de edema, contracturas cicatriciales, particularmente microstomía $^{1,6}$. Una hoja de laringoscopio recta puede tener un rol en facilitar la laringoscopia de un paciente con microstomía ${ }^{3}$. Un conductor de tubo endotraqueal (TET) o un gum elastic bougie pueden facilitar la IOT $^{2}$.

También se ha descrito un mayor porcentaje de éxito de la IOT con el uso de videolaringoscopio versus laringoscopia directa ${ }^{12,13}$. Además, el videolaringoscopio permite evaluar la anatomía del hipofarinx y de la glotis, lo que es particularmente útil si se sospecha injuria inhalatoria ${ }^{2}$.

Para disminuir arcadas (y eventual laringoespasmo) durante la intubación con $\mathrm{FBC}$, se sugiere usar lidocaína en nebulización o gárgaras (técnicas preinducción) o bien instilación directa en spray (postinducción) ${ }^{2}$. Otra técnica usada para facilitar la FBC es la tracción de la lengua (para movilizarla anteriormente) ya sea manualmente con una gasa o con un catéter de aspiración (descrito con una presión de $120 \mathrm{mmHg}$ ), a fin de hacer más expedito el paso del FBC más allá del hipofarin $\mathrm{x}^{4}$. También se puede asistir el paso del FBC a través de una máscara laríngea (ML) de intubación, e incluso a través de una ML corriente ${ }^{2}$.

La ML se ha usado con éxito en pacientes quemados $^{2}$, particularmente pediátricos, demostrando ser un dispositivo seguro y eficaz ${ }^{11}$. Además, si ya existe daño de la mucosa de la vía aérea superior, la ML ayuda a evitar daño adicional que pudiera ocurrir secundario a la intubación ${ }^{6}$. Obviamente en casos de distorsión importante por cicatrices retráctiles, su posicionamiento es más complejo o incluso imposible; una alternativa, particularmente en casos de contractura mento-esternal importante, es la inserción de una ML con la técnica upside-down y una vez dentro de la cavidad oral se rota en $180^{\circ}$ para luego avanzar hacia la región supraglótica ${ }^{14}$.

La intubación nasotraqueal es una alternativa para los pacientes con microstomía, particularmente con FBC (pues incluso el paso de una pinza McGill en la cavidad oral puede estar limitado), en cuyo caso la limitante es el tamaño de 
TET que se puede montar en el FBC disponible y que es capaz de pasar a través de las fosas nasales del paciente ${ }^{2}$. Gosavi ${ }^{8}$ describe el caso de una niña de 12 años con rigidez severa del cuello en la que debido a la imposibilidad de pasar un TET \# 6,5 (mínimo tamaño posible de enhebrar en su FBC) a través de las fosas nasales de la paciente, usó una fosa nasal para pasar el FBC con el que visualizó las cuerdas y a través de la otra fosa nasal pasó un intercambiador de TET a través del que deslizó un TET \# 6,0.

Otra alternativa es intentar la intubación nasotraqueal ciega, con las limitantes de posicionamiento dadas por retracciones cicatriciales y la posibilidad de sangrado que afecte posteriormente la visibilidad para otras técnicas ${ }^{14}$.

Respecto de las contracturas, el uso de relajantes musculares al bloquear la tracción de los tejidos circundantes suele empeorar la retracción ${ }^{14}$; en el caso que se prevea que el manejo de la vía aérea será complejo, es necesario realizar la inducción anestésica en presencia del cirujano, ya que en algunos casos es preciso realizar una escarotomía previo a la laringoscopia (del cuello, de la comisura bucal, etc.), a fin de ampliar el paso del laringoscopio, videolaringoscopio, FBC, etc.; habitualmente este procedimiento se realiza con anestesia local y/o induciendo/sedando con drogas o dosis que preservan la ventilación espontánea ${ }^{2,6,8}$. En casos de no poder ventilar ni intubar a pesar de haber "liberado" las

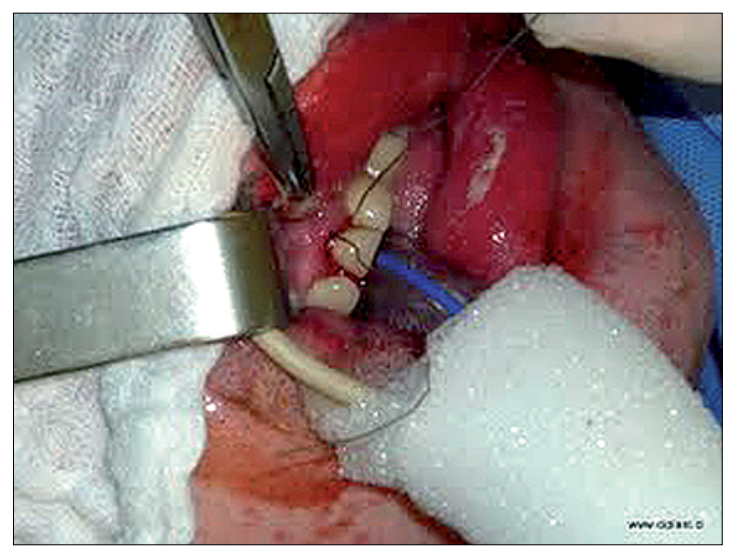

Figura 2. Fijación del TET al diente usando un nudo ballestrinque con una sutura gruesa en un paciente gran quemado. escaras o cicatrices, será necesario realizar una traqueostomía (TQT) por un cirujano experimentado. La TQT también puede realizarse de forma electiva, con sedoanalgesia y/o anestesia local o con anestesia general ${ }^{3}$, sin embargo, la TQT temprana no ha impactado los resultados (dependencia del ventilador, neumonía, sobrevida) y se asocia a riesgos como estenosis subglótica y fístula traqueoesofágica ${ }^{1}$.

Hay reportes pediátricos de casos que dada la dificultad prevista en el manejo fueron sometidos a ECMO antes de la liberación de cicatrices, a modo de puente, hasta lograr asegurar en forma definitiva la vía aérea ${ }^{10}$.

Una dificultad agregada en los pacientes quemados es la fijación del TET o ML. En un paciente con zonas faciales cruentas, o candidato a cirugía reconstructiva que involucre el rostro no es adecuado usar tela o cinta para fijar el TET pues, macerarían la zona, impedirían parte de la escarectomía o desplazarían los injertos ${ }^{1,6}$. Existen varias alternativas frente a este escenario:

- Fijación al diente: es la técnica más usada en el Servicio de Quemados del Hospital de Urgencias Asistencia Pública (HUAP). Con ello se evita el uso de alambre, que es más traumático. Se prefiere usar suturas gruesas (lino, Vicryl $\left.{ }^{\circledR}\right)$ y usar dientes del maxilar superior. La experiencia nos dice que las fijaciones son más estables si hay al menos 3 dientes y el nudo (ballestrinque) se realiza en el diente del

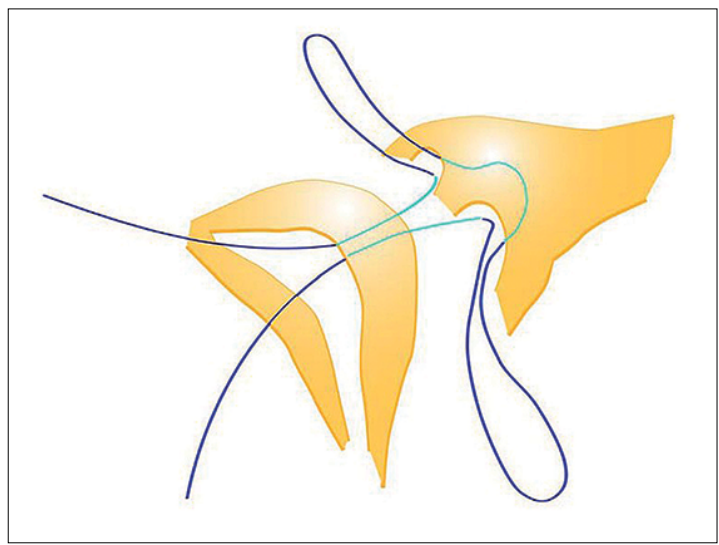

Figura 3. Diagrama del paso de la sutura para la fijación de un TET al tabique nasal (de cartas al editor Fixing the tube en Burns 2011; 37: 177-178). 
medio (Figura 2) ${ }^{7}$.

- Fijación a la encía: en el Servicio de Quemados del HUAP se usa en pacientes edentados, y la realiza el cirujano con suturas gruesas.

- Fijación con sutura desde el sulcus gingival hacia el tabique nasal (Figura 3$)^{5}$.

- Fijación con cinta alrededor del tabique nasal: se introducen 2 sondas, una por cada fosa nasal y se extraen por la boca con una pinza McGill; a cada una se le ata uno de los 2 extremos de una cinta de fijación, luego se traccionan las sondas por la nariz y se extrae la cinta con la que se fija el TET (sale un extremo por cada fosa nasal), teniendo especial cuidado que la úvula no quede atrapada en la cinta durante la tracción (Figura 4$)^{6}$.

Los últimos 2 métodos son útiles en el caso de intubación nasal. También se ha descrito el uso de un pack oral para dar mayor estabilidad al TET 5 .

Finalmente, en los casos en los que la cabeza y cuello formarán parte del campo quirúrgico, es importante alejar y/o aislar el TET y el circuito respiratorio del área estéril. Una alternativa es suspenderlos hacia arriba (Figura 5).

\section{Estrategias ventilatorias}

Existen múltiples razones que tornan a los pacientes quemados en un grupo de difícil manejo ventilatorio. La presencia de quemaduras torácicas, de injuria inhalatoria, el fluid creep (por su impacto directo a nivel pulmonar y/o indirectamente por el efecto restrictivo sobre la caja torácica del síndrome compartamental abdominal asociado al fluid creep), la neumonía, la sepsis, el síndrome de distress respiratorio del adulto, presente en más de la mitad de los pacientes quemados (y su subtipo TRALI, del inglés transfusion related acute lung injury), el daño inducido por el ventilador, la politransfusión con la eventual sobrecarga de volumen que ello pudiese desencadenar, fenómeno también conocido como TACO (del inglés tranfusion asociated cardiac overload), etc. Todos ellos se manifiestan entre otros con laringoespasmo, broncoespasmo, bronquitis, shunt y disminución de la compliance $e^{1,6,15}$. Por lo previamente descrito, en un alto porcentaje de pacientes aplica el uso de estrategias ventilatorias protectoras y estrategias de prevención

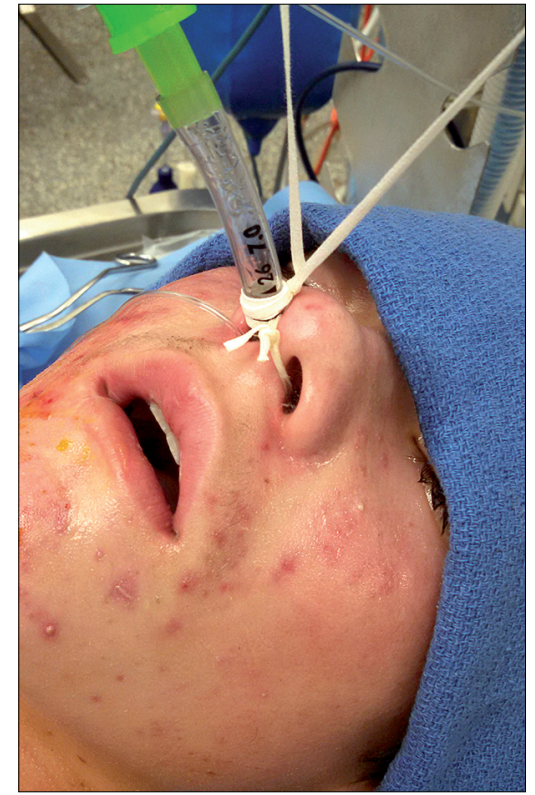

Figura 4. Paciente quemado intubado por nariz. El TET fue sujeto con un amarre septal (Gentileza Dr. Woodson, Shriners Burn Hospital en Galveston, Texas).



Figura 5. "Campo de cabeza" usado en cirugía de cabeza y cuello en los pacientes grandes quemados. Se ata con tela adhesiva al circuito todo aquello que pudiera contaminar el campo: balón de control del TET, sonda nasoyeyunal, termómetro, etc. y luego se envuelve con material estéril (en el HUAP se usa moltopren). 
Desafíos del intraoperatorio - M. Ávila Á. et al.

Tabla 1. Protocolo de prevención de neumonía asociada a ventilación mecánica (NAVM), traducido de American Burn Association Practice Guidelines for Prevention, Diagnosis, and Treatment of Ventilator-Associated Pneumonia (VAP) in Burn Patients. J Burn Care Res 2009; 30: 910-928

\begin{tabular}{|ll|}
\hline Estrategias de prevención de NAVM & Nivel de evidencia \\
\hline $\begin{array}{l}\text { Evitar intubación y re-intubación innecesaria: de ser necesario preferir ambientes controlados } \\
\text { (pabellón quirúrgico, UPC) }\end{array}$ & Clase I \\
$\begin{array}{l}\text { Implementar protocolos de sedación y de destete de la VM } \\
\text { Uso de TET especializados: impregnados de plata; disminuyen la adhesión bacteriana y la formación } \\
\text { de biofilm }\end{array}$ & Clase I \\
$\begin{array}{l}\text { Uso de antisepsia oral con clorhexidina } \\
\text { Uso de TET y SNG por vía oral }\end{array}$ & Clase I \\
$\begin{array}{l}\text { Succión continua de secreciones supraglóticas } \\
\text { Semisentar a los pacientes con nutrición enteral }\end{array}$ & Clase II \\
$\begin{array}{l}\text { Evitar la transfusión de glóbulos rojos } \\
\text { Intercambiadores de humedad-calor para evitar la condensación de los circuitos; vaciar condensado } \\
\text { de los circuitos; y evitar cambios innecesarios de circuitos: los condensados se pueden contaminar con } \\
\text { secreciones de los pacientes y ello puede contaminar la vía aérea baja durante los cambios de posición }\end{array}$ & Clase I \\
$\begin{array}{l}\text { Medidas de control de infecciones y vigilancia de las UPC } \\
\text { Clase I }\end{array}$ & Clase I I \\
\hline
\end{tabular}

$\mathrm{TET}=$ tubo endotraqueal; $\mathrm{SNG}=$ sonda nasogástrica; $\mathrm{UPC}=$ unidad de paciente crítico.

de neumonía asociada a la ventilación (Tabla $1)^{34}$. Concretamente, se aconseja limitar las presiones plateau a $<35 \mathrm{~cm} \mathrm{H}_{2} \mathrm{O}$, usar PEEP suficiente para evitar colapso alveolar y disminuir el volumen corriente; la consecuente disminución en el volumen minuto generará algún grado de hipercapnia permisiva ${ }^{36}$, lo que se observa frecuentemente dado el estado hipermetabólico de los pacientes quemados ${ }^{20}$. La hipercapnia es una consecuencia aceptada, y más aún, se le adjudica efectos antiinflamatorios ${ }^{37}$. Durante las maniobras de reclutamiento, se aceptan por períodos acotados presiones plateau mayores a $35 \mathrm{cms}$ $\mathrm{H}_{2} \mathrm{O}^{37}$. Estas maniobras pueden ser particularmente útiles luego del traslado a pabellón de los pacientes en ventilación mecánica (VM).

Las metas de la VM protectora apuntan a disminuir el daño causado por la VM, y son más factibles de alcanzar asociando terapias broncodilatadoras, aspiración frecuente y en algunos casos broncoscopia terapéutica, siendo el intraoperatorio un buen momento para su ejecución al encontrarse el paciente anestesiado y además, porque en muchos casos va asociado al manejo de la vía aérea (luego de asegurar la vía aérea se procede al examen del árbol traqueobronquial a través del TET), particularmente en pacientes portadores de injuria inhalatoria ${ }^{6,35}$.
En algunos casos la falla respiratoria severa es tal que amerita el uso de modos ventilatorios no convencionales (no disponibles en la máquina de anestesia), en dicho caso será necesario llevar al paciente a pabellón con el ventilador y administrar una técnica anestésica endovenosa ${ }^{1,6,38}$.

\section{Monitorización}

Como en todo paciente crítico, los quemados serán monitorizados de acuerdo a la extensión de sus lesiones, a su estado fisiológico, a la cirugía planeada y al conocimiento de cada sistema de monitorización por parte del equipo médico ${ }^{6,17}$.

La evaluación clínica no puede estar ausente; evaluar parámetros básicos como signos vitales, llene capilar son de gran utilidad para una aproximación inicial al paciente quemado. Pero, considerando los altos niveles de estrés que ocurren en una primera etapa, el monitoreo del paciente quemado debe ser multivariable ya que parámetros como frecuencia cardiaca y presión arterial pueden llevar a confusión en un ambiente adrenérgico exacerbado. Por otra parte, los volúmenes usados en la fórmula de Parkland (sistema de reanimación más difundido en estos pacientes), pueden sobreestimar los requerimientos reales y 
de esta forma evolucionar a un aumento del líquido intersticial, fenómeno conocido como fluid creep, otra razón para monitorizar los volúmenes en forma multivariada ${ }^{31}$.

Los estándares ASA exigen monitorizar a todos los pacientes que van a sedación o procedimientos anestésicos ${ }^{16}$, sin embargo, el paciente quemado puede ofrecer dificultades particulares desde la simple monitorización básica, tales como:

- La simple adhesión de electrodos estándares con gel, particularmente en quemaduras del tronco y extremidades superiores, donde electrodos tipo aguja o la instalación con corchetes (grapas) de sutura podrían ser necesa$\operatorname{rios}^{1,6,18}$, lo que obviamente debe realizarse con el paciente anestesiado (Figura 6).

- No disponer de superficies intactas para la utilización de pulsioxímetros, ya sea por quemadura, edema o por formar parte del campo quirúrgico. En muchos casos se requiere ir rotando el saturómetro de acuerdo a la zona que quede libre (que no forme parte del campo quirúrgico) o buscar zonas alternativas de instalación: lóbulo de la oreja, nariz, labios, lengua ${ }^{20,21}$. Además, la eventual presencia de carboxihemoglobina puede dar lecturas falsamente elevadas, en cuyo caso se hace necesario el uso de un CO-oxímetro ${ }^{1,6}$. Adicionalmente, la curva de pletismografía puede verse alterada en el paciente quemado por la presencia de hipotermia, hipovolemia, disminución del gasto cardiaco y vasconstricción periférica por la elevada adrenergia ${ }^{8}$. En esos casos también se ha recomendado el uso de saturómetro reflectante, que tiene la ventaja de utilizarse en superficies planas ${ }^{6}$; incluso se han elaborado sondas de instalación esofágica con esta tecnología, en donde la perfusión suele estar preservada ${ }^{19}$, pero su uso en clínica ha sido escaso $^{6}$.

- La colocación del manguito de presión arterial no invasiva puede ser un problema cuando hay edema significativo, o quemaduras generalizadas en extremidades. Se deberá instalar sobre gasas estériles y considerar la instalación de catéteres arteriales, los que pueden instalarse sobre zonas quemadas si fuere necesario ${ }^{1,6}$.

En relación al monitoreo invasivo de los pacientes quemados, en las guías de práctica clínica

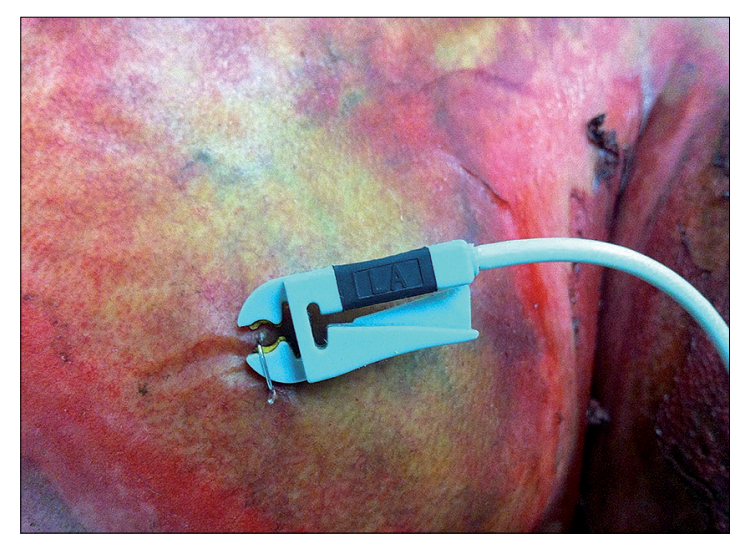

Figura 6. Paciente gran quemado del HUAP con cable caimán de electrocardiografía conectado a corchete quirúrgico.

de la ABA (del inglés American Burn Association) de 2001 se sugiere invadir sólo a los pacientes portadores de shock refractario o con reserva cardiopulmonar limitada ${ }^{22}$. Entre los sistemas de monitoreo más difundidos en la actualidad están:

- Diuresis. La eficacia de la perfusión podrá evaluarse a través de un catéter urinario, donde una diuresis sobre $0,5 \mathrm{ml} \bullet \mathrm{kg}^{-1} \bullet \mathrm{h}^{-1}$ en adultos sigue siendo un buen indicador de gasto cardiaco adecuado, salvo en el caso de los pacientes con quemaduras eléctricas en donde se apunta a conseguir diuresis de al menos 1 $\mathrm{ml} \cdot \mathrm{kg}^{-1} \bullet \mathrm{h}^{-1} 30$.

- Lactato. Si bien los gases arteriales a través de $\mathrm{pH}$, exceso de base y bicarbonato, son pruebas clásicas y con amplio estudio en su utilidad para pacientes en estado de shock, es el lactato sérico el que actualmente ha demostrado una asociación específica con resultados primarios en pacientes quemados. El lactato elevado al ingreso, o incrementos en las primeras $48 \mathrm{~h}$, ha demostrado ser un factor independiente de aumento de mortalidad y morbilidad en pacientes severamente quema$\operatorname{dos}^{32}$.

- Termodilución. El catéter de arteria pulmonar (CAP) se ha utilizado extensamente para la monitorización hemodinámica del paciente críticamente enfermo durante más de dos décadas y ha sido considerado el estándar de oro para el monitoreo de la precarga del ventrículo izquierdo. Sin embargo, la rutina de cateterismo cardiaco derecho con un CAP 
es un proceso que consume tiempo, requiere especial formación para su instalación e interpretación y se asocia con una serie de complicaciones. Por lo demás, el uso del CAP no ha demostrado mejorar la mortalidad o morbilidad en pacientes críticos ${ }^{23,24}$.

- Derivados de la onda de pulso. La medición del contorno de la onda de pulso predice el flujo vascular arterial a través de una interpretación de la onda de presión arterial y el volumen sistólico. Si bien los sistemas de análisis de curva de la presión arterial no han cambiado los resultados de mortalidad en pacientes críticamente enfermos con lesiones por quemaduras, sin duda son técnicas menos invasivas y costosas que la instalación de catéteres de arteria pulmonar, por lo que podrían ser una alternativa efectiva en este tipo de pacientes para el monitoreo de variables volumétricas así como de gasto cardiaco ${ }^{25,26}$. Los tres dispositivos derivados de la onda de pulso más usados son el sistema PICCO (Alemania), el sistema LIDCO (Inglaterra) y el sistema FloTrac Vigileo (USA, Edwards). Cada uno de ellos presenta virtudes y deficiencias sobre el otro; la literatura muestra diversos resultados ${ }^{17}$. Se requieren estudios en quemados con mayor número de pacientes para recomendar su uso rutinario, ya que actualmente se extrapolan datos estudiados en pacientes críticos en general.

- Ecocardiografía transesofágica (ETE). La ETE es también una alternativa para la vigilancia hemodinámica a corto plazo en pacientes con lesiones por quemaduras significativas y con alto impacto hemodinámico ${ }^{25}$. El monitoreo de las tendencias de la contractilidad pueden proporcionar mejor información para la reanimación que indicadores de precarga o volumétricos, ya que aparentemente la depresión miocárdica es un factor tan importante como la hipovolemia en este particular estado de shock ${ }^{27}$. Tradicionalmente la ETE se ha usado por períodos breves y clínicamente ha aportado información diversa, destacando su uso en situaciones de hipotensión de causa no explicada ${ }^{28}$. Sin embargo, hasta la fecha no existen estudios aleatorizados controlados que evalúen el impacto de la ETE en la reanimación del paciente quemado.

- Temperatura central. Para medir la tempera- tura central o core se debería instalar el termómetro, por ejemplo, en el esófago distal o en la arteria pulmonar; ante la dificultad que ello implica, se acepta medir "cerca del core", siendo en ese caso sitios alternativos la boca, el recto, la vejiga, etc. ${ }^{57}$. La medición de temperatura en el intraoperatorio es crítica; un gran quemado puede perder hasta $1{ }^{\circ} \mathrm{C}$ cada $15 \mathrm{~min}$ si no se instalan medidas de calentamiento apropiadas ${ }^{29}$. Sin embargo, la monitorización de la temperatura no sólo apunta a la pesquisa de la hipotermia; en los pacientes ya injertados, candidatos a cirugía reconstructiva, se describe preservación de la vasoconstricción en los injertos, sin embargo, se deteriora la capacidad de vasodilatación, poniendo a esos pacientes en riesgo de hipertermia $^{6}$.

\section{Técnicas de ahorro de sangre}

En la cirugía de los pacientes quemados suele haber pérdidas sanguíneas importantes e insidiosas. En quemaduras profundas en promedio se pierde $50-120 \mathrm{ml}$ de sangre por \% de área escarectomizada. Hay mayor sangramiento a medida que transcurren los días desde ocurrida la lesión (máximo entre los 7-16 días post injuria) y con la presencia de infección ${ }^{1,20}$. El sangrado también se exacerba en presencia de coagulopatía ${ }^{20}$. Sin embargo, la estimación de las pérdidas hemáticas es difícil de calcular, por lo que en ocasiones se requiere de la determinación de hematocrito intraoperatorio para la toma de decisiones (tranfundir o incluso detener el procedimiento) ${ }^{1}$. Usualmente, no más del $20 \%$ de la superficie corporal quemada (SCQ) es escarectomizada en un tiempo quirúrgico, y se planea que idealmente concluya dentro de $2 \mathrm{~h}^{42,51}$. También hay que considerar que la escarectomía se puede realizar con distintas técnicas, pero los métodos más empleados son dos: escarectomía tangencial y escarectomía a fascia. En el primer caso la escara es removida por capas hasta alcanzar tejido viable y se asocia a un ostensible mayor sangrado ${ }^{42}$, en cambio la escarectomía a fascia, como su nombre lo dice remueve la escara y el tejido subcutáneo hasta la fascia, y se realiza en pacientes inestables y con SCQ extensa, sacrificando lo estético a cambio de mayor rapidez y mayor facilidad en 
la hemostasia ${ }^{51}$.

Existen varias técnicas orientadas a disminuir el sangrado intraoperatorio, entre las más usadas está la aplicación tópica (compresas empapadas, spray) y/o inyección de adrenalina bajo el área a escarectomizar o del área que servirá como zona donante. Estudios muestran que si bien es impredecible el impacto hemodinámico de éstas, pareciera que en general no es clínicamente significativo, y que es independiente de la dosis usada ${ }^{33}$. En el Servicio de Quemados del HUAP se usa epinefrina en concentraciones de 1-2 $\mu \mathrm{g} \bullet \mathrm{ml}^{-1}$, limitando el volumen de esta solución a un litro durante un acto quirúrgico. En el caso de la infiltración subgaleal que es usada en los pacientes cuya zona dadora es el cuero cabelludo, y en muchas ocasiones de forma repetida al tratarse de pacientes con grandes superficies quemadas, se usan concentraciones de epinefrina de $0,5 \mu \mathrm{g} \bullet \mathrm{ml}^{-1}$, pues si bien sangran mucho, particularmente después de la primera toma, hay escasos reportes de su impacto hemodinámico (reporte personal del Dr. Jorge Villegas).

Otros vasocontrictores inyectados localmente con este fin son la fenilefrina y los análogos de vasopresina $^{1,6}$.

Otras técnicas descritas son: aplicación de torniquetes a las extremidades, administración de trombina tópica, administración sistémica de vasopresina (con la ventaja de reducir selectivamente el flujo a la piel), administración sistémica de factor VII recombinante activado, o en último caso, realizar el procedimiento en etapas ${ }^{1,6}$.

\section{Inmunodepresión}

La respuesta inflamatoria inicial que se desarrolla en un gran quemado causa una variedad de respuestas hemodinámicas, metabólicas e inmunológicas que pueden desencadenar falla de distintos sistemas ${ }^{39,42}$; sin embargo, una vez superada la etapa inicial de resucitación, los sobrevivientes pueden presentar lo que algunos autores han llamado CARS (del inglés counter anti-inflamatory response syndrome), que sería iniciado por el paciente quemado en un esfuerzo por minimizar el daño tisular inducido por la respuesta inflamatoria ${ }^{39}$. El CARS, junto con la pérdida de piel, la presencia de tejido necrótico (que actúa como "caldo de cultivo" para la co- lonización y proliferación de microorganismos), los requerimientos de procedimientos invasivos (cateterizaciones vasculares y urinaria, ventilación mecánica, etc.) y el aumento de la permeabilidad gastrointestinal sitúa a los pacientes quemados en un estado de particular vulnerabilidad frente a las infecciones ${ }^{39,41,42,44}$.

La respuesta del huésped contra la infección puede clasificarse en inmunidad innata e inmunidad adaptativa. La inmunidad innata actúa inmediatamente; en cambio la inmunidad adaptativa frecuentemente toma más tiempo, pero es más eficiente $^{42}$. El CARS afecta a ambos componentes de la inmunidad ${ }^{39,42}$.

La predisposición a infecciones se vincula positivamente con la extensión de superficie quemada y con su profundidad. También se describe un aumento vinculado con la presencia de injuria inhalatoria y edades extremas. Los pacientes con lesiones autoinferidas, los obesos y diabéticos también son más suceptibles a las infecciones $^{39,42}$.

En materias de inmunomodulación se ha probado tanto a nivel experimental como clínico numerosas estrategias:

- Inmunonutrición: en donde destaca el aporte precoz de alimentación enteral, por sobre la parenteral, ya que logra disminuir la colonización de las zonas quemadas por flora intestinal, y la suplementación del aminoácido glutamina, ambas promisorias ${ }^{44-46}$.

- Descontaminación selectiva del tracto digestivo: como una forma de combatir la traslocación bacteriana aumentada a nivel intestinal y subsecuente colonización con bacilos gramnegativos, sin embargo, esta estrategia no es recomendable por el riesgo de aumentar la resistencia bacteriana ${ }^{47}$.

- Otras técnicas con menor validación: vacunación, con la limitante de proteger contra organismos únicos; modulación de citokinas, tanto aporte exógeno (interferón $\gamma$ ) como neutralización, con resultados poco alentadores, sumados a potenciales efectos adversos; estimulación de la hematopoiesis, administración de factor estimulante de colonias de granulocitos y de granulocitos/macrófagos (G-SCF y GM-SCF respectivamente), cuyo impacto clínico aún no ha sido establecido ${ }^{39}$.

No se ha logrado avanzar lo suficiente en ma- 
terias de inmunomodulación; si a ello sumamos la creciente resistencia bacteriana, las infecciones han llegado a constituir la principal causa de muerte en esta población ${ }^{40,42,43}$.

El principal objetivo de la escarectomía precoz es detener la tremenda respuesta inflamatoria que desencadena y de ese modo prevenir la infección al cerrar temporal o definitivamente las zonas cruentas. De ese modo se restaura la función de barrera de la piel ${ }^{42,49}$. Ser un facilitador para que el paciente sea intervenido precozmente (dentro de 5 días luego de ocurrida la lesión, apenas se estabilice al paciente) es un aporte fundamental del anestesiólogo.

Puede ser dificultoso determinar qué pacientes quemados están infectados, puesto que la taquicardia, la taquipnea, la trombocitosis y la fiebre son muy frecuentes en esta población ${ }^{15}$.

En promedio toma 7 días desde la injuria para que se presente bacteremia. La primera semana son principalmente grampositivos y posteriormente gramnegativos. La bacteremia se asocia a un aumento de 4 veces la mortalidad. Se sugiere que el manejo vaya orientado a la prevención más que a nuevos métodos de detección ${ }^{49}$. Es por lo anterior que en los grandes quemados se sugiere el uso de "aislamiento protector" ${ }^{50}$ sumado a un meticuloso cuidado respiratorio y de los accesos vasculares, el cual no debe discontinuarse durante el intraoperatorio ${ }^{15}$.

El aislamiento protector o inverso considera estricta higiene de manos, uso de ropa estéril, y uso universal de gorro, mascarilla, guantes y bata desechable por parte del equipo de trabajo cada vez que se interactúa con el paciente (Figura 7$)^{50}$.

Los cuidados respiratorios fueron resumidos en la tabla de prevención de NAVM de la ABA (Tabla 1$)^{47}$ y los cuidados de los accesos vasculares se detallarán en la sección Accesos vasculares de este mismo artículo.

\section{Hipotermia}

La termoregulación posee un componente aferente, mecanismos regulatorios centrales y un componente eferente.

Cabe destacar que la percepción no se altera en los pacientes quemados, dado que la aferencia proveniente de la piel da cuenta de sólo 5-20\% de la información que llega a los centros reguladores ${ }^{6}$.

El control de la temperatura central es muy fino, por ello el rango en que se mantiene es estrecho $\left(36,6-37,5^{\circ} \mathrm{C}\right)$. La percepción de cambios de $0,2^{\circ} \mathrm{C}$ determinan respuestas por parte del centro regulador. Sin embargo, luego de sufrir una quemadura este set point se ve modificado debido al estado hipermetabólico y a la produc-

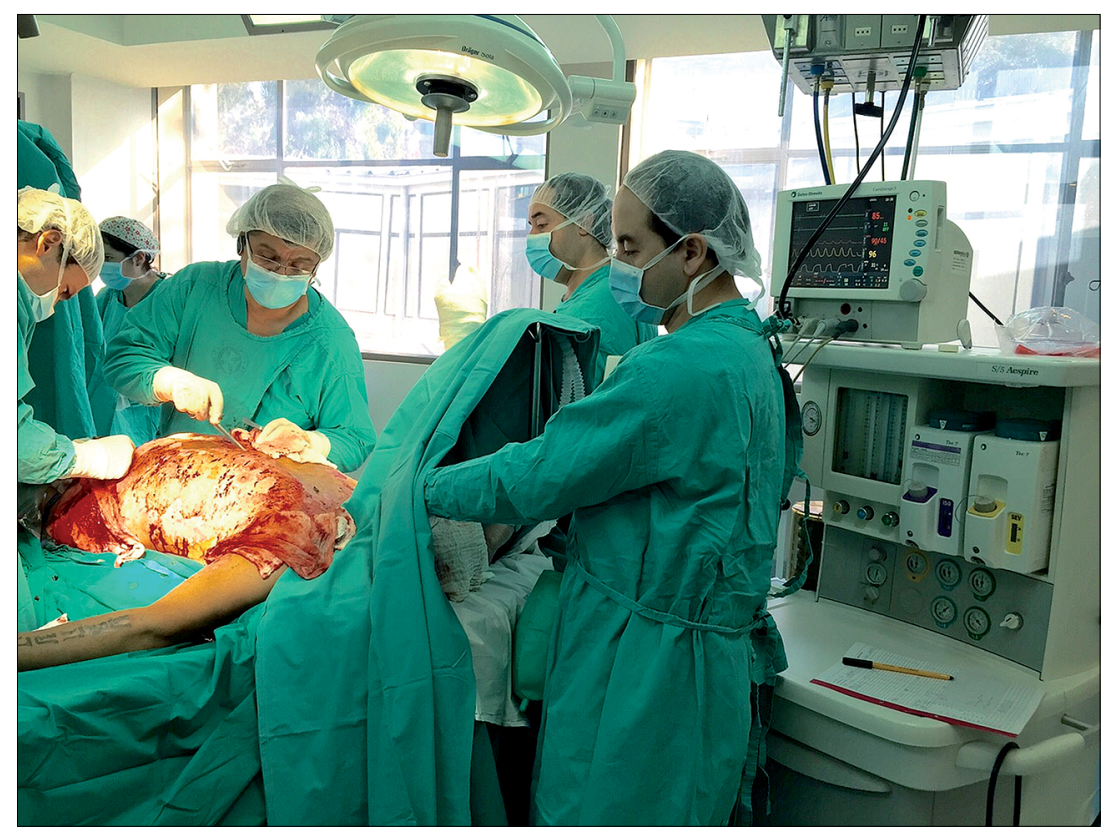

Figura 7. Equipo quirúgico-anestésico aplicando los principios del aislamiento protector en un paciente gran quemado (Servicio de quemados, HUAP). 
ción de mediadores inflamatorios vinculados a pirogénesis ${ }^{1}$. Está descrito un aumento de $0,03^{\circ} \mathrm{C}$ por $\%$ de $\mathrm{SCQ}^{52}$.

Las respuestas eferentes se refieren fundamentalmente a comportamientos voluntarios, tales como el abrigo, la búsqueda de sitios cálidos, el aumento de la ingesta (de comidas preferentemente calientes) que en los pacientes quemados están limitados (sedación, vendaje de las extremidades, etc. $)^{6}$.

La hipotermia es una de las principales limitantes en la cirugía de los grandes quemados. Sus mecanismos de producción son múltiples. Los quemados pierden calor a su entorno a una elevada tasa debido a la falta de dermis y epidermis. A ello se suma la incapacidad de la microcirculación para regular los cambios en la temperatura ambiente, pues se pierde la capacidad vasoconstrictora en las zonas quemadas y escarectomizadas, especialmente con la escarectomía a fascia $^{6,15}$. Otro mecanismo de pérdida de calor es la evaporación que se exacerba frente a la pérdida de la piel, y que en algunos pacientes puede llegar a $4.000 \mathrm{ml} \cdot \mathrm{m}^{-2}$ de SCQ al día ${ }^{6,53}$. Las pérdidas son aún más pronunciadas en los niños, debido a su mayor proporción superficie/volumen ${ }^{15,53}$. Los pacientes geriátricos también son más susceptibles a la hipotermia debido al deterioro de la circulación periférica, menor eficiencia de los centros reguladores, menor capacidad de generar calofríos y menor grasa corporal ${ }^{56}$.

La hipotermia se produce durante el traslado al pabellón quirúrgico, durante los preparativos quirúrgicos cuando los vendajes son removidos, durante la infusión de hemoderivados o cristaloides y/o coloides a baja temperatura sumado a un pabellón quirúrgico frío.

Los pacientes anestesiados tienen en parte abolidos los mecanismos de respuesta a la hipotermia (vasoconstricción, calofríos y termogénesis no relacionada con calofríos) y además pierden la protección de los comportamientos voluntarios (brazo eferente), por ende, la responsabilidad de monitorizar y mantener una temperatura adecuada es del anestesiólogo $0^{6}$. Esto es particulamente importante en los pacientes quemados dado el mayor set point ya descrito en esta población, pues conlleva a una respuesta de estrés e hipermetabolismo adicional con el consecuente impacto en la evolución del paciente (retraso en la cicatrización, aumento en la tasa de infecciones, retardo en la rehabilitación, etc. $)^{55}$.

Complicaciones asociadas a la hipotermia del paciente quemado son: coagulopatía, con el consiguiente aumento del sangrado intraoperatorio y de los requerimientos tranfusionales; metabolismo alterado de drogas (incluyendo anestésicos); isquemia miocárdica y arritmias; aumento de infecciones de la zona operatoria; calofríos y un aumento significativo de la morbi-mortalidad. Un ejemplo es la injuria pulmonar aguda o ALI (del inglés Acute Lung Injury), que tras la disminución de tan sólo $1^{\circ} \mathrm{C}$ aumenta significativamente en los pacientes quemados sometidos a cirugía ${ }^{29,54}$.

La hipotermia detectada en el preoperatorio debe ser manejada previo al traslado al pabellón quirúrgico ${ }^{6}$. Es más efectivo prevenir la hipotermia a través del "precalentamiento", el cual permite disminuir la gradiente temperatura central/ temperatura periférica y de ese modo disminuir la hipotermia por redistribución ${ }^{57}$. Ello se puede concretar con el uso de frazadas de calentamiento con aire forzado que han mostrado ser muy efectivas ${ }^{58}$.

Respecto del intraoperatorio, es preciso llevar la temperatura del pabellón por sobre $25^{\circ} \mathrm{C}$, evitar exposición de superficies quemadas, usar coberturas plásticas que pueden disminuir la pérdida por evaporación, usar sueros y hemoderivados tibios (usualmente a $38^{\circ} \mathrm{C}$ ) y entibiar los gases inspirados $\left(41^{\circ} \mathrm{C}\right)^{6,15,57}$.

\section{Accesos vasculares}

Se recomienda insertar vías venosas periféricas cortas y gruesas en territorio no quemado, sin embargo, durante el shock inicial su instalación se hace muy difícil debido a la vasoconstricción; posteriormente la dificultad la constituyen las cicatrices $^{6,15,55}$. Cuando las extremidades están quemadas (o multipuncionadas debido a necesidad de múltiples cirugías) se hace necesario instalar catéteres venosos centrales $(\mathrm{CVC})^{6,55}$. Muchas veces no queda alternativa que instalarlos en áreas quemadas, en cuyo caso la fijación debe asegurarse que se realiza en tejido vital, por tanto en quemaduras profundas amerita debridar la escara previo a la sutura (Figura 8$)^{6}$. Sin duda, la instalación ecoguiada de los CVC debe considerarse en un grupo con significativas distorsiones anatómicas secundarias a edema o cicatrices, 


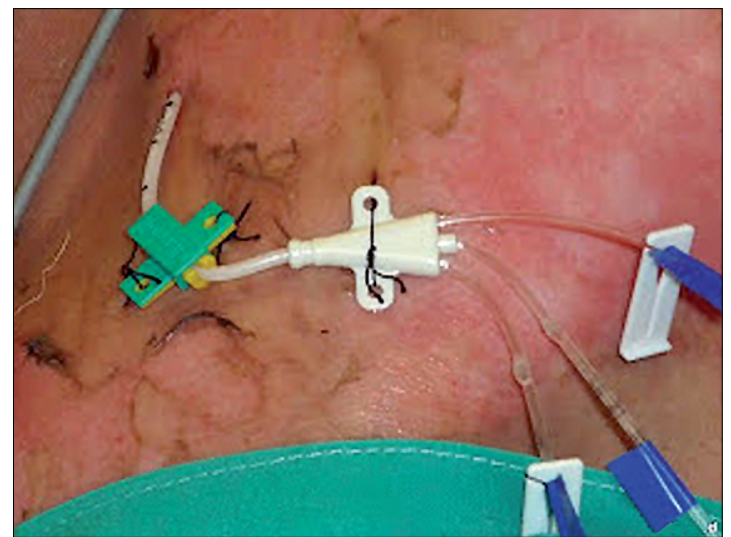

Figura 8. CVC instalado sobre piel quemada.

aunque teniendo presente que la calidad de las imágenes puede ser afectada por la presencia de cicatrices $^{6}$.

Respecto de los CVC hay amplio debate en relación con el sitio ideal de inserción (en el entendido que cerca o a través de áreas quemadas aumenta la posibilidad de colonización), el tiempo de permanencia in situ y la conveniencia de cambio por técnica de Seldinger (en vez de puncionar un nuevo sitio). Lo cierto es que en el subgrupo de pacientes quemados críticos, hay escasos estudios de buena calidad, siendo la gran mayoría, antiguos, con muestras pequeñas, no controlados (o con controles históricos) y sometidos a análisis sin ajuste por confundentes. Estudios recientes avalarían el uso de CVC cubiertos de antibióticos ${ }^{49}$.

En el Servicio de Quemados del HUAP no se realizan cambios programados de $\mathrm{CVC}$, si no de acuerdo a criterios clínicos (presencia de signos de infección del sitio de inserción o en presencia de un proceso séptico que no se pueda explicar por otro foco). Los cambios son a un nuevo sitio y para la prevención de infecciones se aplican las recomendaciones de la $\mathrm{ASA}^{59}$ : técnica asép- tica (lavado quirúrgico de manos), uso de gorro, mascarilla, guantes estériles, delantal estéril y cobertura estéril alrededor del sitio de inserción; el lavado y pincelado de la zona es con solución de clorhexidina alcohólica.

\section{Extubación}

En el postoperatorio inmediato la decisión de extubación es multifactorial. Habitualmente se indica VM postoperatoria en pacientes hemodinámicamente inestables (que han requerido gran aporte de volumen y/o drogas vasoactivas en dosis altas), hipotérmicos, pacientes con injerto laminar de cara y cuello (especialmente pacientes con historial de psicopatía y/o dificultad en lograr sedación adecuada) o pacientes con vía aérea difícil ${ }^{1,6}$, sin embargo en este último grupo una alternativa es extubar dejando in situ un intercambiador de tubo a través del cual se puede oxigenar y deslizar otro TET de ser necesario ${ }^{60}$.

En los pacientes con injerto en la región del dorso es criterio del anestesiólogo (de acuerdo con el resto del equipo) la decisión de extubar o dejar en VM. En el primer caso, el procedimiento debe ser realizado en prono, por lo cual es necesario realizarlo con el paciente despierto, en el entendido de que si fuera necesario dar presión positiva en el caso de un laringoespasmo por ejemplo, el colocar al paciente en posición supina, pone en riesgo la permanencia in situ de los injertos.

En los pacientes previamente ventilados habitualmente se les mantiene en VM, sin embargo, de acuerdo con el equipo de intensivistas, pacientes en condiciones de destete y particularmente con historia de vía aérea difícil, pueden ser extubados en pabellón una vez finalizada la cirugía, pues en ese lugar el anestesiólogo contará con condiciones óptimas en caso de requerir reintubar ${ }^{47}$.

\section{Referencias}

1. Kaiser HE, Kim CM, Sharar SR et al. Advances in Perioperative and Critical Care of the Burn Patient. Anesthesia Management of Major Thermal Burn Injuries in Adults. Adv Anesth 2013;31(1):137-161.
2. Cochran A. Inhalation injury and endotracheal intubation. J Burn Care Res 2009 JanFeb;30(1):190-191.

3. Caruso TJ, Janik LS, Fuzaylov G. Airway management of recovered pediatric patients with severe head and neck burns: a review. Paediatr Anaesth 2012
May;22(5):462-468.

4. Haastrup AA, Méndez P, Cote CJ. Suction the tongue: a new adjunct for improving the laryngeal view for fiberoptic intubation. Anesth Analg 2011 Jun;112(6):1512-1513.

5. Wong DS, Chiu T, Liu LH et al. Fixing the tube. Burns 2011 Feb;37(1):177-178. 
6. David N. Herndon. Total Burn

Care: Expert Consult - Online and Print. Chapter 14: Anesthesia for burned patients. Lee C. Woodson, Edward R. Sherwood, Asle Aarsland, Mark Talon, Michael P. Kinsky, Elise M. Morvant.

7. Yadav R, Solanki SL. Fixation of ProSeal ${ }^{\mathrm{TM}}$ laryngeal mask airway in a child with facial burns. Burns 2013 Feb;39(1):187-188.

8. Gosavi KS, Nirkhi AM. Exchange catheter-assisted fiberoptic nasal intubation: a modified technique. Anesth Analg 2011 Jul;113(1):205-206.

9. Embu HY, Yiltok SJ, Isamade ES. Anaesthetic management of mentosternal contractures where resources are limited. Niger J Med 2008 Apr-Jun;17(2):143-145.

10. Sheridan RL, Ryan DP, Fuzaylov $\mathrm{G}$ et al. Case records of the Massachusetts General Hospital. Case 2-2008. Case 5-2008. An 18-month-old girl with an advanced neck contracture after a burn. N Engl J Med 2008 Feb;358(7):729-735.

11. McCall JE, Fischer CG, Schomaker E et al. Laryngeal mask airway use in children with acute burns: intraoperative airway management. Paediatr Anaesth 1999;9(6):515-520.

12. Woo CH, Kim SH, Park JY et al. Macintosh laryngoscope vs Pentax-AWS video laryngoscope: comparison of efficacy and cardiovascular responses to tracheal intubation in major burn patients. Korean J Anesthesiol 2012 Feb;62(2):119-124.

13. Esnault $P$, Prunet $B$, Cotte $J$ et al. Tracheal intubation difficulties in the setting of face and neck burns: myth or reality? Am J Emerg Med 2014 Oct;32(10):1174-1178.

14. Han TH, Teissler H, Han RJ, Gaines JD et al. Managing difficult airway in patients with post-burn mentosternal and circumoral scar contractures. Int J Burns Trauma
2012;2(2):80-85

15. Anderson TA, Fuzaylov G. Perioperative anesthesia management of the burn patient. Surg Clin North Am. 2014; 94: n851-61

16. American Society of Anesthesiologists. 2011. Standards for Basic Anesthetic Monitoring. Accesado en http://www.asahq.org/ /media/ For\%20Members/documents/ Standards $\% 20$ Guidelines $\% 20$ Stmts/Basic\%20Anesthetic\%20 Monitoring\%202011.ashx el 30 marzo de 2015.

17. Lavrentieva A, Palmieri T. Determination of cardiovascular parameters in burn patients using arterial waveform analysis: a review. Burns 2011 Mar;37(2):196-202.

18. Sofos SS, Tehrani H, Shokrollahi K et al. Surgical staple as a transcutaneous transducer for ECG electrodes in burnt skin: safe surgical monitoring in major burns. Burns 2013 Jun;39(4):818819.

19. Pal SK, Kyriacou PA, Kumaran S et al. Evaluation of oesophageal reflectance pulse oximetry in major burns patients. Burns 2005 May;31(3):337-341.

20. Anaesthesia and intensive care for major burns. Continuing Education in Anaesthesia. Critical Care \& Pain 2012;12:118-122.

21. Coté CJ, Daniels AL, Connolly M et al. Tongue oximetry in children with extensive thermal injury: comparison with peripheral oximetry. Can J Anaesth 1992 May;39(5 Pt 1):454-457.

22. American Burn Association. Practice guidelines for burn care. J Burn Care Rehabil 2001;22:S1S67.

23. Shah MR, Hasselblad V, Stevenson LW et al. Impact of the pulmonary artery catheter in critically ill patients: meta-analysis of randomized clinical trials. JAMA 2005 Oct;294(13):1664-1670.

24. Takala J. The pulmonary artery catheter: the tool versus treatments based on the tool. Crit Care 2006;10(4):162.

25. Berton C, Cholley B. Equipment review: new techniques for cardiac output measurement-oesophageal Doppler, Fick principle using carbon dioxide, and pulse contour analysis. Crit Care 2002 Jun;6(3):216-221.

26. Lavrentieva A, Palmieri T. Determination of cardiovascular parameters in burn patients using arterial waveform analysis: a review. Burns 2011 Mar;37(2):196-202.

27. Wang GY, Ma B, Tang HT, et al. Esophageal echo-Doppler monitoring in burn shock resuscitation: are hemodynamic variables the critical standard guiding fluid therapy? J Trauma 2008 Dec;65(6):1396-1401.

28. Etherington L, Saffle J, Cochran A. Use of transesophageal echocardiography in burns:a retrospective review. J Burn Care Res 2010 Jan-Feb;31(1):36-39.

29. Oda J, Kasai K, Noborio M et al. Hypothermia during burn surgery and postoperative acute lung injury in extensively burned patients. J Trauma 2009 Jun;66(6):1525-1529.

30. Greenhalgh DG. Burn resuscitation: the results of the ISBI/ABA survey. Burns 2010 Mar;36(2):176-182.

31. Pruitt BA Jr. Protection from excessive resuscitation: "pushing the pendulum back". J Trauma 2000 Sep;49(3):567-568.

32. Cochran A, Edelman LS, Saffle JR et al. The relationship of serum lactate and base deficit in burn patients to mortality. J Burn Care Res 2007 MarApr;28(2):231-240.

33. Cartotto R, Kadikar N, Musgrave MA et al. What are the acute cardiovascular effects of subcutaneous and topical epinephrine for hemostasis during burn surgery? 
J Burn Care Rehabil 2003 SepOct;24(5):297-305.

34. Mosier MJ, Pham TN. American Burn Association Practice guidelines for prevention, diagnosis, and treatment of ventilator-associated pneumonia (VAP) in burn patients. J Burn Care Res 2009 Nov-Dec;30(6):910-928.

35. Mosier MJ, Pham TN, Park DR et al. Predictive value of bronchoscopy in assessing the severity of inhalation injury. J Burn Care Res 2012 Jan-Feb;33(1):65-73.

36. Peck MD, Koppelman T. Lowtidal-volume ventilation as a strategy to reduce ventilatorassociated injury in ALI and ARDS. J Burn Care Res 2009 Jan-Feb;30(1):172-175.

37. Dries DJ. Key questions in ventilator management of the burn-injured patient (first of two parts). J Burn Care Res 2009 JanFeb;30(1):128-138.

38. Dries DJ. Key questions in ventilator management of the burn-injured patient (second of two parts). J Burn Care Res 2009 Mar-Apr;30(2):211-220.

39. David N. Herndon. Total Burn Care: Expert Consult - Online and Print. Chapter 22: The immunological response and strategies for intervention Erle D. Murphey, Edward R. Sherwood, Tracy Toliver-Kinsky.

40. Alp E, Coruh A, Gunay GK et al. Risk factors for nosocomial infection and mortality in burn patients: 10 years of experience at a university hospital. J Burn Care Res 2012 MayJun;33(3):379-385.

41. Barbut F, Yezli S, Mimoun M et al. Reducing the spread of Acinetobacter baumannii and methicillin-resistant Staphylococcus aureus on a burns unit through the intervention of an infection control bundle. Burns 2013 May;39(3):395-403.

42. Church D, Elsayed S, Reid O et al. Burn wound infections.

Clin Microbiol Rev 2006

Apr;19(2):403-434.

43. Rajput A, Saxena R, Singh KP et al. Prevalence and antibiotic resistance pattern of metallobeta-lactamase-producing Pseudomonas aeruginosa from burn patients-experience of an Indian tertiary care hospital. J Burn Care Res 2010 MarApr;31(2):264-268.

44. Peng YZ, Yuan ZQ, Xiao GX. Effects of early enteral feeding on the prevention of enterogenic infection in severely burned patients. Burns 2001 Mar;27(2):145-149.

45. Lam NN, Tien NG, Khoa CM. Early enteral feeding for burned patients-an effective method which should be encouraged in developing countries. Burns 2008 Mar;34(2):192-196.

46. Tan HB, Danilla S, Murray A et al. Immunonutrition as an adjuvant therapy for burns. Cochrane Database Syst Rev 2014;12:CD007174.

47. Mosier MJ, Pham TN. American Burn Association Practice guidelines for prevention, diagnosis, and treatment of ventilator-associated pneumonia (VAP) in burn patients. J Burn Care Res 2009 Nov-Dec;30(6):910-928.

48. Lloyd JR, Hight DW. Early laminar excision: improved control of burn wound sepsis by partial dermatome debridement. J Pediatr Surg 1978 Dec;13(6 6D):698-706.

49. Wolf SE, Arnoldo BD. The year in burns 2012. Burns 2013 Dec;39(8):1501-1513.

50. Ministerio de Salud. Normas de aislamiento y Manual de Procedimientos. Minsal; 1988.

51. Ministerio de Salud. Guía Clínica Gran Quemado. Minsal; 2007.

52. Caldwell FT Jr, Wallace BH, Cone JB. The effect of wound management on the interaction of burn size, heat production, and rectal temperature. J Burn Care Rehabil 1994 MarApr;15(2):121-129.

53. Fuzaylov G, Fidkowski CW. Anesthetic considerations for major burn injury in pediatric patients. Paediatr Anaesth 2009 Mar;19(3):202-211.

54. Oda J, Kasai K, Noborio M et al. Hypothermia during burn surgery and postoperative acute lung injury in extensively burned patients. J Trauma 2009 Jun;66(6):1525-1529.

55. Atiyeh BS, Gunn SW, Dibo SA. Nutritional and pharmacological modulation of the metabolic response of severely burned patients: review of the literature (part 1). Ann Burns Fire Disasters 2008 Jun;21(2):63-72.

56. David N. Herndon. Total Burn Care: Expert Consult - Online and Print. Chapter 36: Care of geriatric patients. Porro LJ, Demling RH, Pereira CT, Herndon DN.

57. Hooper VD, Chard R, Clifford T et al. ASPAN's Evidence-Based Clinical Practice Guideline for the Promotion of Perioperativec Normothermia: Second Edition. Journal of PeriAnesthesia Nursing 2010; 25 (6): 346-365.

58. Andrzejowski J, Hoyle J, Eapen $\mathrm{G}$ et al. Effect of prewarming on post-induction core temperature and the incidence of inadvertent perioperative hypothermia in patients undergoing general anaesthesia. Br J Anaesth 2008 Nov;101(5):627-631.

59. A Report by the American Society of Anesthesiologists Task Force on Central Venous Access. Practice Guidelines for Central Venous Access. Anesthesiology 2012;116(3):539-573.

60. Prakash S, Mullick P. Airway management in patients with burn contractures of the neck [en prensa]. Burns 2015 Apr;S0305-4179(15)00089-3. 